\title{
Erratum to: A fast VLSI architecture of a hierarchical block matching algorithm for motion estimation
}

\author{
Kausik Ghosh • Anindya Sundar Dhar
}

Published online: 8 March 2013

(c) Springer-Verlag Berlin Heidelberg 2013

\section{Erratum to: J Real-Time Image Proc}

DOI 10.1007/s11554-012-0300-7

Unfortunately, the article note was incorrectly published in the original publication of the article. The correct article note should read as:

"This work was carried out as a part of Master degree program and it does not represent any opinion or position of his present employer."

The online version of the original article can be found under doi: 10.1007/s11554-012-0300-7.

K. Ghosh $(\bowtie)$. A. S. Dhar Department of Electronics and EC Engineering, Indian Institute of Technology, Kharagpur 721302,

West Bengal, India

e-mail: 09ec6212@iitkgp.ac.in

A. S. Dhar

e-mail: asd@ece.iitkgp.ernet.in 